\begin{tabular}{|c|l|}
\hline Title & $\begin{array}{l}\text { Endoscopic nasobiliary drainage is the most suitable preoperative biliary drainage method in the management of } \\
\text { patients with hilar cholangiocarcinoma }\end{array}$ \\
\hline Author(s) & $\begin{array}{l}\text { Kawakami, Hiroshi; Kuwatani, Masaki; Onodera, Manabu; Haba, Shin; Eto, Kazunori; Ehira, Nobuy uki; Y amato, } \\
\text { Hiroaki; Kudo, Taiki; T anaka, Eiichi; Hirano, Satoshi; Kondo, Satoshi; A saka, Masahiro }\end{array}$ \\
\hline Citation & $\begin{array}{l}\text { JOURNAL OF GA ST ROENTEROLOGY, 46(2), 242-248 } \\
\text { https://doi.org/L0.1007/300535-010-0298-1 }\end{array}$ \\
\hline Issue Date & 2011-02 \\
\hline Doc URL & http://hdl.handle.net/2115/44960 \\
\hline Rights & The original publication is available at www.springerlink.com \\
\hline Type & article (author version) \\
\hline File Information & Kawakami46-2_242-248.pdf \\
\hline
\end{tabular}

Instructions for use 
Title page

\section{Endoscopic nasobiliary drainage is the most suitable preoperative biliary drainage method in the management of patients with hilar cholangiocarcinoma}

Short title: Biliary drainage for hilar cholangiocarcinoma

Hiroshi Kawakami, ${ }^{1}$; Masaki Kuwatani, ${ }^{1}$; Manabu Onodera, ${ }^{1}$, Shin Haba, ${ }^{1} ;$ Kazunori

Eto, ${ }^{1} ;$ Nobuyuki Ehira, ${ }^{1}$; Hiroaki Yamato, ${ }^{1}$; Taiki Kudo, ${ }^{1}$; Eiichi Tanaka, ${ }^{2}$; Satoshi Hirano, ${ }^{2}$; Satoshi Kondo, ${ }^{2}$; Masahiro Asaka, ${ }^{1}$

${ }^{1}$ Department of Gastroenterology, Hokkaido University Graduate School of Medicine, Sapporo, Japan

${ }^{2}$ Department of Surgical Oncology, Hokkaido University Graduate School of Medicine, Sapporo, Japan

Address correspondence to: Hiroshi Kawakami, MD, PhD

Department of Gastroenterology, Hokkaido University Graduate School of Medicine,

Kita 15, Nishi 7, Kita-ku, Sapporo 060-8638, Japan

Tel: +81117161161 (Ext 5920); Fax: +81 117067867

E-mail: hiropon@med.hokudai.ac.jp (H. Kawakami) 


\section{Abstract}

Background: Controversy exists over the preferred technique of preoperative biliary drainage $(\mathrm{PBD})$ in patients with hilar cholangiocarcinoma (HCA). The goal of this retrospective study was to identify the preferred technique of PBD for HCA.

Methods: A total of 128 consecutive patients with HCA diagnosed between September 1999 and December 2009 who underwent PBD were included in this study. The study compared outcomes of endoscopic nasobiliary drainage (ENBD), endoscopic biliary stenting (EBS), and percutaneous transhepatic biliary drainage (PTBD) in patients with HCA.

Results: There were no significant differences in preoperative laboratory data, rates of major hepatectomy, or decompression periods among the 3 groups. Complications were significantly more frequent in the EBS group compared with either the ENBD or PTBD group $(p<0.05)$. Drainage tube occlusion with cholangitis was significantly more common in the EBS group compared with either the ENBD or PTBD group $(p<0.0001)$. Patients in the PTBD group experienced serious complications including vascular injury (8\%) and cancer dissemination (4\%). Patients in the ENBD and EBS groups had mild post-endoscopic retrograde cholangiopancreatography pancreatitis (5\%). Conversion procedures were significantly more common in the EBS group compared with the ENBD and PTBD groups $(p<0.05)$. There was no significant difference in postsurgical morbidity or mortality among the 3 groups.

Conclusions: Drainage tube occlusion with cholangitis was a frequent complication associated with EBS. PTBD was associated with serious complications such as vascular injury and cancer dissemination. ENBD was found to be the most suitable method for initial PBD management in patients with HCA. 
Kawakami-3

\section{Keywords}

Hilar cholangiocarcinoma; Preoperative biliary drainage; Endoscopic biliary drainage;

Endoscopic nasobiliary drainage; Percutaneous transhepatic biliary drainage 


\section{Abbreviations}

PTBD, Percutaneous transhepatic biliary drainage

PBD, Preoperative biliary drainage

ENBD, Endoscopic nasobiliary drainage

EBS, Endoscopic biliary stenting

ERCP, Endoscopic retrograde cholangiopancreatography

ICG, Indocyanine green

EBD, Endoscopic biliary drainage

EST, Endoscopic sphincterotomy

HCA, Hilar cholangiocarcinoma 


\section{Introduction}

Percutaneous biliary drainage (PTBD) has been commonly performed as a preferred technique of preoperative biliarydrainage (PBD) in patients with hilar cholangiocarcinoma (HCA). In recent years, remarkable advances in the development of endoscopic technologies have resulted in the widespread use of endoscopic devices. In particular, an endoscopic biliary drainage (EBD) method has been increasingly applied in the clinical setting, and several groups have reported its utility $[1,2]$. However, only a small number of comparative studies of EBD and PTBD have been performed. Recent reports suggest that EBD requires more procedures and has a higher risk of cholangitis complications caused by occlusion in endoscopic biliary stents compared with PTBD [3]. Additionally, a consensus on the proper application of PBD has not been reached because no randomized controlled trial has been conducted to determine the utility of PBD in treating patients with HCA.

In Japan, guidelines for the management of biliary tract carcinomas [4] provide equal preference to PTBD, EBD [endoscopic nasobiliary drainage (ENBD), and endoscopic biliary stenting (EBS)], and surgical drainage approaches for performing PBD. These guidelines also recommend methods that can be safely performed with the equipment and techniques available at each facility. However, PTBD is an invasive procedure associated with several complications. Early complications include tube dislocation, hemobilia, hepatic artery pseudoaneurysm, hepatic artery bile duct fistula, and injury and occlusion of the portal vein, and late complications include catheter tract implantation metastasis caused by bile leakage $[5]$.

EBD consists of two categories: external biliary drainage such as ENBD 
and internal biliary drainage such as EBS. To date, there has been no clear consensus on which of these methods is more suitable for EBD. In addition, there has been no report that compares PTBD with either ENBD or EBS as PBD methods.

We retrospectively studied the clinical outcomes of PBD in patients who had had a laparotomy to treat HCA and we compared PTBD, ENBD, and EBS to determine which technique was the most suitable.

\section{Patients and methods}

We enrolled a total of 128 consecutive patients who had undergone PBD (at various hospitals) before surgical resection of HCAs was carried out at Hokkaido University Hospital (Department of Gastroenterology and Surgical Oncology) between September 1999 and December 2009. Patients who had no biliary drainage $(n=14)$ or biliary drainage combined with ENBD plus EBS $(n=4)$ were excluded from this study. Patient characteristics were as follows: the median age was 70 years (range 44-84 years), and 96 men (75\%) and 32 women were (25\%) enrolled. All patients with PBD followed by surgical resection were examined in the present study.

The lesions were classified according to the Bismuth classification as follows: type I, no obstruction of the confluence of the right and left hepatic ducts; type II, limited obstruction of the confluence; type IIIa or IIIb, extended obstruction to the right or left ductal ramifications; and type IV, extended bilateral obstruction [6].

In accordance with the Declaration of Helsinki, written informed consent was obtained from each patient or his or her family members before endoscopic retrograde cholangiopancreatography $(\mathrm{ERCP})$.

Surgical resection was indicated when distant metastases were absent. 
Planned hilar resections included resection of the hilar plate with or without hemihepatectomy, as well as caudate lobectomy. A right hepatectomy and caudate lobectomy with extrahepatic bile duct resection were indicated for Bismuth type I, II, and IIla tumors. A left hepatectomy and caudate lobectomy with extrahepatic bile duct resection were indicated for Bismuth type Illb tumors. A right or left trisectionectomy and caudate lobectomy with extrahepatic bile duct resection were indicated for Bismuth type IV tumors [5, 7, 8]. Portal vein or arterial reconstruction was performed even when resectable vascular invasion (portal vein or artery) existed $[7,8]$. There were no inoperable cases due to problems in biliary drainage during the study.

At our hospital (Department of Gastroenterology and Surgical Oncology), the initial biliary drainage method for HCA was usually unilateral ENBD for the future remnant liver. Only 12 patients (9.4\%) with HCAs had the initial biliary drainage procedures performed at our hospital. In this study, we categorized patients who had received PBD, including those with referrals, into 3 groups. Patients were categorized as being in the ENBD, EBS, or PTBD group according to the drainage method that was initially applied. Major hepatectomy included right or left trisectionectomy, right or left hepatectomy including caudate lobectomy, or more extensive hepatectomy. Preoperative laboratory data, including total serum bilirubin level, indocyanine green (ICG) at $15 \mathrm{~min}$, the decompression period required to reach normal total bilirubin levels, conversion to biliary drainage, complications, and operative morbidity and mortality were evaluated according to the initial biliary drainage method.

\section{Preoperative biliary drainage}


All the PTBD procedures were performed under ultrasound guidance. The diameter of placement tubes was generally $7-10 \mathrm{Fr}$. However, the diameters were 12-16 Fr for cases involving percutaneous transhepatic cholangioscopy. PTBD was utilized in our department whenever the performance of ENBD was considered difficult. Indwelling tubes for EBD were inserted either by the physicians that the patients consulted initially or by clinical staff in our department. The physicians consulted initially used a catheter for contrast imaging for bile duct cannulation during ERCP. If the bile duct could not be cannulated using a catheter, pancreatic guidewire placement was attempted in most cases. The tube diameter for ENBD was $7 \mathrm{Fr}$. A straight-a or reverse-a type tube was most commonly used and a pigtail-type tube was used in some cases. EBS was performed only by the physicians consulted initially. For EBS cases, the tube diameter was 7 or $8.5 \mathrm{Fr}$ and a straight type tube was cannulated. In some hospitals, endoscopic sphincterotomy (EST) was performed for EBS regardless of tube diameter.

However, we used a conventional catheter method or wire-guided cannulation for endoscopic bile duct cannulation. All patients underwent ENBD using a 5- to 7-Fr plastic ENBD tube (Olympus Medical Systems, Tokyo, Japan). When patients developed cholangitis with catheter obstruction after ENBD or EBS, we either exchanged the catheter immediately or inserted an additional ENBD catheter with EST to the other lobe upon viewing the segmental cholangitis after computed tomography findings. All endoscopic procedures were performed by 2 endoscopists (H.K., M.K.) while the patient was under conscious sedation with intravenous pethidine hydrochloride and diazepam. Intravenous antibiotics were administered after ERCP in all patients. Neither selective cannulation of common bile ducts with 
precutting nor pancreatic stenting for preventing post-ERCP pancreatitis was performed.

As this study was a retrospective review of cases, consent from the patient or their family was required, and the institutional review board of our institution approved the study protocols (Hokkaido University Hospital, Clinical research approved No. 010-0010).

\section{Evaluations}

When a patient was undergoing biliary drainage at the initial hospital, the date of biliary drainage, diameter of the biliary drainage catheter, date of complication, and therapy used for the complication were checked and recorded for each patient. An interview survey of the initial doctors was conducted from April 2004 to December 2009.

\section{Definition of each parameter}

In this study, jaundice was defined as a serum total bilirubin level greater than $2.0 \mathrm{mg} / \mathrm{dL}$. The preoperative maximum value of the serum total bilirubin level was recorded before biliary drainage. The minimum ICG at 15 min was recorded immediately before surgery. Catheter obstruction was defined when there was high fever (more than $38^{\circ} \mathrm{C}$ ) and elevated serum hepatobiliary enzymes that improved after exchange of the catheter. Segmental cholangitis was defined as a case in which no catheter obstruction was evident and no improvement occurred after exchange of the catheter. Contralateral cholangitis was defined as a case that occurred in an undrained area. Accidents of ERCP were classified using the 
classification of Cotton et al. [9]. Based on the consensus meeting held in 1991, the diagnostic criteria for post-ERCP pancreatitis were abdominal pain lasting $>24 \mathrm{~h}$ after ERCP and hyperamylasemia ( $>3$ times the upper limit of the normalrange). The Cotton classification was generally used for the assessment of severity, with the exception that time until first food consumption was used rather than duration of hospitalization. The other techniques followed the original classification. Postsurgical mortality included all in-hospital deaths. All postsurgical complications that lengthened the hospital stay were considered as cases of operative morbidity. Postoperative hepatic insufficiency was defined as a rise in serum total bilirubin concentration, with a total bilirubin level greater than $10.0 \mathrm{mg} / \mathrm{dL}$ during the postoperative period.

We examined complications in each group based on the rates of complications observed before converting to or adding other drainage methods. We also thoroughly investigated the characteristics of the complications associated with each method. A case in which other drainage methods had to be used was described as converting biliary drainage. Cases in which a catheter was exchanged for one of the same type or in which additional ones were placed were not considered converting biliary drainage cases.

\section{Statistical analysis}

Statistical analysis was performed using Dr. SPSS for Windows, version 8.0.1 J (SPSS, Chicago, IL, USA). A test of independence and Tukey's multiple comparison test were performed to compare age, sex, Bismuth classification [6], drained area, rates of major hepatectomy, complication rates, characteristics of 
complications, conversion rates of $\mathrm{PBD}$, and operative morbidity and mortality among the groups. The maximum levels of serum total bilirubin, the minimum ICG at $15 \mathrm{~min}$ immediately before surgery, and the decompression period required to reach normal total bilirubin levels (less than $2.0 \mathrm{mg} / \mathrm{dL}$ ) were compared among the 3 groups by using one-way analysis of variance. Comparison of major complications was performed using the Fisher exact probability test. A $p<0.05$ was considered statistically significant.

\section{Results}

\section{Baseline patient characteristics}

There were no inoperable cases due to problems in biliary drainage in the ENBD, EBS, or PTBD groups during the study. No significant differences were found in median age $(71,70,71$ years) or sex distribution (male/female: $44 / 16,16 / 4$, 36/12) among the 3 groups. Additionally, no significant differences were found in the rates of major hepatectomy $(78.3,80.0,79.2 \%)$ among the groups. No significant differences were found in Bismuth classification among the three groups [Type I, II, IIla, IIlb, IV: 12/16/12/8/12 (ENBD group, $n=60$ ), 3/6/4/3/4 (EBS group, $n=20$ ), 4/12/8/8/16 (PTBD group, $n=48$ )]. No significant differences were found in drainage area among the three groups [unilateral, bilateral: 57/3 (ENBD group, $n=60), 15 / 5$ (EBS group, $n=20$ ), 35/13 (PTBD group, $n=48$ )]. The numbers and types of ENBD tubes placed in patients in the study were as follows: single tube in 57 patients; double tubes in 3 patients (all patients with bilateral drainage). The numbers and types of EBS tubes were as follows: single tube in 15 patients; double tubes in 5 patients (all patients with bilateral drainage). The numbers and types of PTBD tubes 
were as follows: single tube in 28 patients (all patients with unilateral drainage); double tubes in 20 patients.

No significant differences were found in maximum value of total bilirubin $(p=0.300)$, preoperative ICG retention at 15 min before operation $(p=0.092)$, or the decompression period required to reach normal total bilirubin levels $(p=0.793)$ among the 3 groups (Table 1).

\section{Complications of preoperative biliary drainage}

Table 2 shows the rates and characteristics of complications of PBD. The rates of complications were $38.3 \%(23 / 60), 65.0 \%(13 / 20)$, and $31.3 \%(15 / 48)$ in the ENBD, EBS, and PTBD groups, respectively. The rate of complications in the EBS group was significantly higher than that in the PTBD group $(p<0.05)$ and that in the EBS group was marginally higher than that in the ENBD group $(p<0.01)$. Duplicate cases were included in each group in regard to the characteristics of complications.

Among minor complications, it was especially notable that a significantly higher rate of tube occlusion was found in the EBS group than in either the ENBD or PTBD group $(p<0.0001)$. Tube occlusions in all 3 groups were associated with acute obstructive cholangitis, but re-cannulation of ENBD alleviated the occlusions in the EBS and ENBD groups. A single case of tube occlusion in the PTBD group was successfully resolved by cleaning the tube and administering an antibacterial drug. On the other hand, no significant differences were found among the 3 groups in rates of tube dislocation or contralateral segmental cholangitis. In the ENBD group, 3 out of 6 cases with contralateral segmental cholangitis were found after ENBD tube imaging. In all EBD groups (including the ENBD and EBS groups), 3 cases of post-ERCP 
pancreatitis were found; these cases were minor and resolved successfully with conservative therapy.

As a major complication, a single case of retroperitoneal perforation associated with EST was found in the ENBD group, but it was quickly resolved by conservative therapy. In the PTBD group, 4 cases involving injury of the portal vein and 3 cases of cancer dissemination were observed. Among these 7 cases, treatment procedures had to be converted in 4 cases (Table 2).

\section{Conversion rates of preoperative biliary drainage}

As shown in Table 3, conversion rates of PBD were 21.7\%(13/60), 95.0\% (19/20), and $4.2 \%(2 / 48)$ in the ENBD, EBS, and PTBD groups, respectively. The rate in the EBS group was significantly higher than that in either the ENBD or PTBD group $(p<0.05)$. Table 3 also shows the characteristics of the PBD conversions. In the ENBD group, 7 cases were converted to EBS, of which 6 cases included elective treatment with EBS by the physicians consulted initially. In the EBS group, 18 cases were converted to ENBD. Of these conversions, the majority (66.7\%; $12 / 18$ cases) were cases of tube occlusion with cholangitis and 4 cases involved conversion to ENBD for the purpose of a staging diagnosis.

Postsurgical morbidity and mortality

Table 4 shows the characteristics of morbidity and mortality. Morbidity rates were $10.0 \%(6 / 60), 5.0 \%(1 / 20)$, and $18.5 \%(9 / 48)$ in the ENBD, EBS, and PTBD groups, respectively, and no significant differences among these rates were found. Mortality rates were $0 \%(0 / 60), 5.0 \%(1 / 20)$, and $6.3 \%(3 / 48)$ in the ENBD, EBS, and PTBD 
groups, respectively, and no significant differences were found among these rates, either.

\section{Discussion}

Discussion

In the present retrospective study, no significant difference was found in the preoperative decompression period required to reach normal total bilirubin levels among the ENBD, EBS, and PTBD groups. However, the rate of complications was significantly higher in the EBS group $(p<0.05)$ compared with either of the other two groups. In particular, tube occlusion was found in $60 \%$ of EBS cases, representing a significantly higher rate and suggesting that this complication is a serious concern associated with EBS. According to an analysis limited to EBS occlusion, the median duration of occlusion was 27.5 days (range: 9-57 days), which was a relatively short duration (data not shown). In the present study, all cases of EBS occlusion could be treated by conversion to ENBD. However, when patients developed fever after EBS placement, differential diagnosis was difficult. Specifically, determining whether the fever was due to cholangitis in the drainage area or due to contralateral segmental cholangitis in an undrained area was difficult, because EBS is an internal fistula treatment and bile color or output cannot be checked. In contrast to the problem with differential diagnosis for EBS, this type of differential diagnosis can be easy for ENBD, in which bile color and output can be checked.

It has been reported that cholangitis is a risk factor for hepatic insufficiency after major hepatic resection [10-12]. Sakata et al. [12] conducted a study involving 81 patients with HCA who underwent major hepatic resection, and 
they evaluated the effects of preoperative cholangitis on inhospital mortality in those patients. Sakata's group found that mortality was higher in patients with cholangitis than in those without this complication (33 vs. $6 \%$ ) and that cholangitis was the only independent variable relating to postoperative in-hospital mortality, according to a multivariate analysis.

In the present retrospective study, there was no case in which a converting procedure was required due to cholangitis associated with tube occlusion in EBS or a decline in residual liver function. However, in combination with results reported by other groups [10-12], it should be noted that EBS, which has a higher rate of cholangitis due to tube occlusion, should be avoided for PBD to treat HCA. On the other hand, tube dislocations were commonly found in the ENBD group for tubes that did not have an a-loop shape (data not shown). These dislocations are presumably caused by the peristaltic movements of the stomach and duodenum or by the passage of food through these organs. Thus, the placement of ENBD tubes must be performed carefully. Furthermore, it is important to examine the tube placement in ENBD with an X-ray examination of the abdomen to check for any dislocation, as well as to examine bile color and output. Because ENBD tubes are easy to replace, we found in this study that occluded or dislocated tubes could be replaced without causing any serious complications in all cases. However, it should be noted that 3 out of 6 cases of contralateral segmental cholangitis were found after ENBD tube imaging, which was performed to carefully examine the bile duct. Additional PTBD tubes are commonly placed to treat contralateral segmental cholangitis, but recently the placement of two ENBD tubes through a transpapillary approach has become available. Arakura et al. [2] performed a single ENBD 
procedure in the remnant lobe of the liver in 62 patients with HCA, and found that this approach was effective in $74 \%$ of the patients. The remaining $26 \%$ of the patients required additional drainage with ENBD, and half of these patients were successfully treated with a transpapillary approach. In the present study, we placed additional ENBD tubes, without altering the drainage route, upon EST median incisions in 3 of 6 patients with contralateral segmental cholangitis in the ENBD group. These patients, who had been treated since 2006, quickly recovered with this treatment method. Although there is a possibility that performing EST can cause complications of reflux cholangitis, we have not observed reflux cholangitis in the clinical setting to date, suggesting that EST is acceptable. However, in the event that cholangitis occurs in an undrained area even when two ENBD tubes are placed, we should consider performing additional PTBD because the condition is no longer adequately controlled by ENBD.

Post-ERCP pancreatitis also can be a potential complication associated with EBD. However, the incidence of post-ERCP pancreatitis was permissible (5\%) in the present retrospective study and each case was mild. In the PTBD group, we found cases of injury to the portal vein branches and portal vein occlusion, which required surgery or conversion procedures. Cancer dissemination associated with bile spillage was another complication observed in this group. Even experienced specialists cannot always avoid bile spillage with PTBD, and cancer dissemination should be considered a possible outcome of PTBD. Therefore, PTBD should be performed on a limited basis as an optional procedure when additional EBD is complicated because of bilateral segmental cholangitis.

Limitations of the present study include the retrospective design and the 
evaluation of subjects from a single center. Patients with HCA who required biliary drainage were treated with ENBD at our hospital, and a selection bias could have influenced the outcome. However, the selection bias would have been very limited, because the study included a small number (9.4\%) of patients who had initial biliary drainage performed at our hospital. Larger studies are required to further elucidate the current situation of preoperative biliary drainage for HCA. Future prospective randomized controlled trials using EBD or PTBD will likely be difficult to conduct because of the major complications associated with the placement of PTBD tubes. In conclusion, ENBD is highly recommended as a preferred PBD method for HCA.

\section{Acknowledgments}

The authors declare that there was no grant support, and there are no financial disclosures and no conflicts of interest. 


\section{References}

1. Maguchi H, Takahashi K, Katanuma A, Osanai M, Nakahara K, Matuzaki S, et al. Preoperative biliary drainage for hilar cholangiocarcinoma. J Hepatobiliary Pancreat Surg. 2007;14:441-446.

2. Arakura N, Takayama M, Ozaki Y, Maruyama M, Chou Y, Kodama R, et al. Efficacy of preoperative endoscopic nasobiliary drainage for hilar cholangiocarcinoma. J Hepatobiliary Pancreat Surg. 2009;16:473-477.

3. Kloek JJ, van der Gaag NA, Aziz Y, Rauws EA, van Delden OM, Lameris JS, et al. Endoscopic and Percutaneous Preoperative Biliary Drainage in Patients with Suspected Hilar Cholangiocarcinoma. J Gastrointest Surg. 2010;14:119-125.

4. Nagino M, Takada T, Miyazaki M, Miyakawa S, Tsukada K, Kondo S, et al. Preoperative biliary drainage for biliary tract and ampullary carcinomas. $\mathrm{J}$ Hepatobiliary Pancreat Surg. 2008;15:25-30.

5. Kawakami H, Kuwatani M, Etoh K, Haba S, Yamato H, Shinada K, et al. Endoscopic retrograde cholangiography versus peroral cholangioscopy to evaluate intraepithelial tumor spread in biliary cancer. Endoscopy. 2009;41:959-964.

6. Bismuth $\mathrm{H}$, Nakache R, Diamond T. Management strategies in resection for hilar cholangiocarcinoma. Ann Surg. 1992;215:31-38.

7. Kondo S, Hirano S, Ambo Y, Tanaka E, Okushiba S, Morikawa T, et al. Forty consecutive resections of hilar cholangiocarcinoma with no postoperative mortality and no positive ductal margins: results of a prospective study. Ann Surg. 2004;240:95-101.

8. Hirano S, Kondo S, Tanaka E, Shichinohe T, Tsuchikawa T, Kato K, et al. 
Outcome of surgical treatment of hilar cholangiocarcinoma: a special reference to postoperative morbidity and mortality. J Hepatobiliary Pancreat Surg. 2009 Oct 8. [Epub ahead of print]

9. Cotton PB, Lehman G, Vennes J, Geenen JE, Russell RC, Meyers WC, et al. Endoscopic sphincterotomy complications and their management: an attempt at consensus. Gastrointest Endosc. 1991;37:383-393.

10. Nagino M, Nimura Y, Hayakawa N, Kamiya J, Kondo S, Sasaki R, et al. Logistic regression and discriminant analyses of hepatic failure after liver resection for carcinoma of the biliary tract. World J Surg. 1993;17:250-255.

11. Kanai M, Nimura Y, Kamiya J, Kondo S, Nagino M, Miyachi M, et al. Preoperative intrahepatic segmental cholangitis in patients with advanced carcinoma involving the hepatic hilus. Surgery 1996;119:498-504.

12. Sakata J, Shirai Y, Tsuchiya Y, Wakai T, Nomura T, Hatakeyama K. Preoperative cholangitis independently increases in-hospital mortality after combined major hepatic and bile duct resection for hilar cholangiocarcinoma. Langenbecks Arch Surg. 2009;394:1065-1072. 
Table 1. Baseline characteristics

ENBD group $(n=60)$ EBS group $(n=20)$ PTBD group $(n=48)$

Median age (range)

Gender

\section{Male}

Female

Major hepatectomy

Bismuth-Corlette classification [7]

Type I/II/IIla/IIlb/IV

Drained area

Unilateral/Bilateral

Preoperative laboratory data

T.Bil $>2.0 \mathrm{mg} / \mathrm{dL}$, number of patients
$71(45-81)$

$70(59-77)$

$71(45-81)$

N.S

44

16

$47(78.3 \%)$

$12 / 16 / 12 / 8 / 12$

$3 / 6 / 4 / 3 / 4$

$4 / 12 / 8 / 8 / 16$

N.S

$57 / 3$

$15 / 5$

$35 / 13$

$38(63.3 \%)$

$9(45 \%)$

$36(75 \%)$

N.S 


$\begin{array}{lllll}\text { T.Bil (mg/dL), average [range] } & 9.9[2.2-29.3] & 9.0[2.4-14.7] & 12.0[3-25.3] & \text { N.S } \\ \text { ICG (\%), average [range] } & 21.0[3.5-35] & 13.6[9.9-22.7] & 30.0[3.5-35] & \text { N.S } \\ \text { Decompression period, average (range) } & 11.5[1-134] & 11.9[1-28] & 11.0[1-154] & \text { N.S }\end{array}$

The decompression period was the period required to reach normal total bilirubin levels.

ENBD endoscopic nasobiliary drainage, EBS endoscopic biliary stenting, PTBD percutaneous transhepatic biliary drainage, T.Bil maximum total bilirubin, ICG indocyanine green retention at $15 \mathrm{~min}$, N.S. not significant 
Table 2. Complication rates and characteristics of preoperative biliary drainage

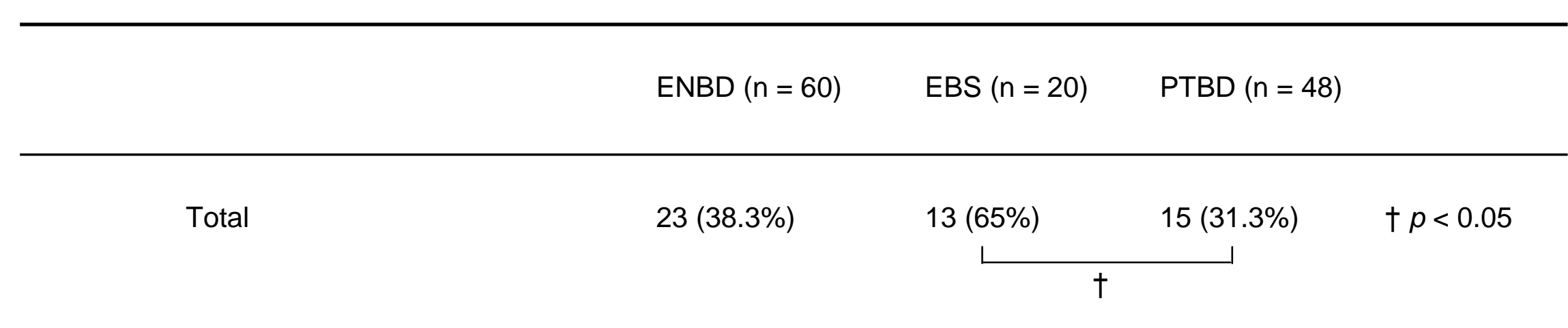

Minor complications

$\begin{array}{lllll}\text { Tube occlusion with cholangitis } & 6(10 \%) & 12(60 \%)^{*} & 1(2.1 \%) & { }^{*} p<0.0001 \\ \text { Tube dislocation } & 9(15 \%) & 1(5 \%) & 7(14.6 \%) & \text { N.S } \\ \text { Contralateral segmental cholangitis } & 6(10 \%) & 1(5 \%) & 4(8.3 \%) & \text { N.S } \\ \text { Post-ERCP pancreatitis } & 2(3.3 \%) & 1(5 \%) & -\end{array}$

Major complications

$\begin{array}{llll}\text { Total } & 1(1.7 \%) \S & - & 7(14.6 \%) \S\end{array} \quad p<0.01 \S$




$\begin{array}{llll}\text { Retroperitoneal perforation } & 1(1.7 \%) & - & - \\ \text { Injury of portal vein } & - & - & 4(8.3 \%) \\ \text { Cancer dissemination } & - & - & 3(6.3 \%)\end{array}$

Numbers in regard to characteristics of preoperative biliary drainage include duplicate cases.

ENBD endoscopic nasobiliary drainage, EBS endoscopic biliary stenting, PTBD percutaneous transhepatic biliary drainage, ERCP endoscopic retrograde cholangiopancreatography, N.S. not significant

† The rate of complications in the EBS group was significantly higher than that in the PTBD group $(p<0.05)$.

* Among minor complications, it was especially notable that a significantly higher rate of tube occlusion was found in the EBS group than in either the ENBD or PTBD group $(p<0.0001)$.

$\S$ Among major complications, the rate of complications in the PTBD group was significantly higher than in the ENBD group $(p<0.01)$. 
Table 3. Converting rates and characteristics of preoperative biliary drainage

\begin{tabular}{|c|c|c|c|c|}
\hline & ENBD group $(n=60)$ & EBS group $(n=20)$ & PTBD group $(n=48)$ & \\
\hline Total & $13(21.7 \%)$ & $19(95 \%)$ & $\begin{array}{c}2(4.17 \%) \\
\end{array}$ & $\dagger p<0.05$ \\
\hline \multicolumn{5}{|c|}{ Converting procedure } \\
\hline ENBD & - & 18 & 1 & \\
\hline EBS & 7 & - & 1 & \\
\hline PTBD & 6 & 1 & - & \\
\hline
\end{tabular}

ENBD endoscopic nasobiliary drainage, EBS endoscopic biliary stenting, PTBD percutaneous transhepatic biliary drainage † The rate of conversion of preoperative biliary drainage in the EBS group was significantly higher than that in the PTBD group $(p<0.05)$. 
Table 4. Postsurgical morbidity and mortality

\begin{tabular}{|c|c|c|c|c|}
\hline & $\begin{array}{l}\text { ENBD group } \\
\qquad(n=60)\end{array}$ & $\begin{array}{l}\text { EBS group } \\
\qquad(n=20)\end{array}$ & $\begin{array}{l}\text { PTBD group } \\
(n=48)\end{array}$ & \\
\hline Total & $6(10 \%)$ & $1(5 \%)$ & $9(18.5 \%)$ & N.S \\
\hline \multicolumn{5}{|l|}{ Infection } \\
\hline Liver abscess & $3^{a}$ & - & 5 & N.S \\
\hline Intra-abdominal abscess & 2 & $1^{\mathrm{a}}$ & $1^{\mathrm{a}}$ & N.S \\
\hline Sepsis & 1 & $1^{\mathrm{a}}$ & $1^{\mathrm{a}}$ & N.S \\
\hline Hepatic insufficiency & $1^{a}$ & $1^{a}$ & 3 & N.S \\
\hline In-hospital death & 0 & 1 & 3 & N.S \\
\hline
\end{tabular}


ENBD endoscopic nasobiliary drainage, EBS endoscopic biliary stenting, PTBD percutaneous transhepatic biliary drainage, N.S. not significant

a Postsurgical morbidity data include duplicate cases 